\title{
Fatores associados e índice de adiposidade corporal (IAC) em adultos: estudo de base populacional
}

\author{
Factors associated with body adiposity index (BAI) in adults: \\ population-based study
}

\author{
Wellington Segheto ${ }^{1}$ \\ Pedro Curi Hallal ${ }^{2}$ \\ João Carlos Bouzas Marins ${ }^{3}$ \\ Danielle Cristina Guimarães da Silva ${ }^{4}$ \\ France Araújo Coelho ${ }^{1}$ \\ Andreia Queiroz Ribeiro ${ }^{1}$ \\ Silvia Helena Oliveira Morais ${ }^{1}$ \\ Giana Zarbato Longo ${ }^{1}$
}

\footnotetext{
${ }^{1}$ Departamento de Nutrição e Saúde, Universidade Federal de Viçosa (UFV). R. P. H. Holfs s/n, Centro. 36570-000 Viçosa MG Brasil.wsegheto@gmail.com ${ }^{2}$ Programa de PósGraduação em Epidemiologia, Universidade Federal de Pelotas. Pelotas RS Brasil. ${ }^{3}$ Departamento de Educação Física e Esporte, UFV. Viçosa MG Brasil. ${ }^{4}$ Centro das Ciências Biológicas e da Saúde, Universidade Federal do Oeste da Bahia. Barreiras BA Brasil.
}

\begin{abstract}
The objective of this study was to analyze factors associated with the body adiposity index. A population-based, cross-sectional study was conducted with 964 adults between 20 and 59 years of age, in Viçosa, State of Minas Gerais, Brazil. Household questionnaires and anthropometric measurements were applied to obtain data. Multiple analysis was performed using linear regression, adjusted for the sampling design effect. The prevalence of excess body adiposity was 36.89 (CI 30.81 to 43.43), being higher among men (48.18\% - CI 39.09 to 57.34). Factors positively associated with body adiposity index were: female sex $(p=0.002)$; age in years $(p<0.001)$; those living with a partner $(p=0.022)$. Self-evaluation of positive health $(p=0.048)$ was negatively associated with the body adiposity index. The prevalence of excess body fat was high and the sex, age, marital status, health self-evaluation and dissatisfaction with body image were determinant factors to the increase in body adiposity in this population, indicating a marked risk for chronic diseases resulting from excess body fat.

Key words Adiposity, Associated factors, Population survey, Adults
\end{abstract}

Resumo Objetivou-se analisar os fatores associados ao indice de adiposidade corporal. Um estudo transversal de base populacional foi conduzido com 964 adultos, de 20 e 59 anos, em Viçosa, MG, Brasil. Aplicou-se questionários domiciliares e medidas antropométricas para se obter os dados. A análise múltipla foi realizada por meio de regressão linear, ajustada pelo efeito do desenho amostral. A prevalência de excesso de adiposidade corporal foi 36,89 (IC 30,81-43,43), sendo mais elevada nos homens $(48,18 \%$ - IC 39,09-57,34). Associou-se positivamente com o indice de adiposidade corporal o sexo feminino ( $p=0,002)$, idade em anos $(p<0,001)$, aqueles que viveram com companheiro $(p=0,022)$ e com insatisfação com a imagem corporal $(p<0,001)$. A autoavaliação da saúde positiva $(p=0,048)$ esteve associada negativamente com o indice de adiposidade corporal. A prevalência de excesso de adiposidade corporal foi elevada, sendo o sexo, a faixa etária, o estado civil, a autoavaliação da saúde e a insatisfação com a imagem corporal fatores determinantes para o aumento da adiposidade corporal, nesta população, indicando um forte risco para doenças crônicas não transmissíveis decorrentes do excesso de adiposidade.

Palavras-chave Adiposidade, Fatores associados, Inquérito populacional, Adultos 


\section{Introdução}

A obesidade está relacionada com o aumento da gordura corporal, a qual pode trazer prejuízos para a saúde ${ }^{1}$ e associa-se a diversas doenças, tais como: as cardiovasculares ${ }^{2}$, o diabetes tipo $2^{3}$, a apneia do sono $\mathrm{o}^{4}$ e o câncer ${ }^{5}$. Essas comorbidades, associadas ao excesso de adiposidade, resultam em maior gasto com a saúde ${ }^{6}$ e levam a um aumento da morbidade e diminuição da expectativa de vida ${ }^{7}$.

Na população norte-americana, no período de 1998 a 2006, os gastos relacionados à obesidade aumentaram de US $\$ 78,5$ bilhões para US $\$ 147$ bilhões $^{8}$ e, no Brasil, identificou-se um aumento de, aproximadamente, $\mathrm{R} \$ 16$ milhões entre 2008 e $2011^{9}$. Essas estimativas reforçam a necessidade da inclusão de medidas que identifiquem o excesso de adiposidade corporal de forma simples e objetiva, permitindo que programas de prevenção e tratamento possam ser implementados para diminuir a prevalência desta morbidade e, consequentemente, os gastos relacionados ao seu tratamento e suas comorbidades.

Neste contexto, diversas técnicas de avaliação da composição corporal estão descritas. Os métodos de avaliação realizados em laboratórios e com equipamentos sofisticados, representam a melhor opção para avaliação da composição corporal ${ }^{10}$, porém são caros e de difícil acesso, principalmente para estudos populacionais. Como alternativa, encontram-se as medidas antropométricas que são simples de serem utilizadas, inócuas e apresentam poucas restrições culturais para sua utilização, tornando essas medidas as mais aplicáveis em grandes grupos populacionais ${ }^{11}$.

Dentre os métodos antropométricos disponíveis, o índice de massa corporal (IMC) é um dos mais utilizadas na prática clínica e em estudos de base populacional. Porém, algumas limitações do IMC estão descritas na literatura, tais como uma possível variação para a mesma faixa de IMC entre indivíduos de uma mesma população e entre diferentes populações ${ }^{12}$, impossibilidade de determinação precisa do IMC em locais os quais a balança não está disponível ${ }^{13}$, em indivíduos de raças/etnia diferentes ${ }^{14} \mathrm{e}$ em atletas com elevada porcentagem de massa magra ${ }^{15}$.

Em função dessas limitações, foi proposto um índice com o objetivo de mensurar a adiposidade corporal de forma simples e objetiva, denominado índice de adiposidade corporal (IAC), calculado a partir da medida da circunferência do quadril e da estatura ${ }^{16}$. A equação proposta para a avaliação da adiposidade corporal pelo IAC foi desenvolvida com dados de adultos americanos descendentes de mexicanos com idades entre 18-67 anos, e testada em uma amostra de africanos-americanos, usando DEXA como método de referência ${ }^{16}$. Observou-se que o IAC teve correlação mais forte com a porcentagem de gordura corporal em comparação com o IMC ${ }^{16}$.

Diversos estudos foram realizados para validação do IAC $^{13,17-19}$ e para verificar a sua capacidade preditora de alterações metabólicas ${ }^{20}$ e cardiovasculares $^{21}$. Na população latino-americana, com adultos de Bogotá, na Colômbia, o IAC foi utilizado para verificar a sua capacidade preditiva de risco cardiovascular ${ }^{21}$, a sua validade em estimar o percentual de gordura corporal ${ }^{21,22}$ e para verificar a sua capacidade em predizer a síndrome metabólica ${ }^{23}$. Além disso, Carpio Rivera et al. ${ }^{24}$ verificaram a capacidade do IAC em predizer o percentual de gordura corporal em estudantes da Costa Rica. Com a população brasileira, foram conduzidos estudos para estimar o risco para o diabetes mellitus tipo $2^{20}$, avaliar a gordura corporal em adultos de ambos os sexos com doença renal crônica ${ }^{25}$ e em adultos com obesidade severa $^{26}$; para verificar a sua validade em mulheres brasileiras na meia-idade ${ }^{27}$ e com mulheres com lipodistrofia ${ }^{17}$. No entanto, não foram encontrados estudos que identificaram a prevalência de excesso de adiposidade corporal em adultos e seus fatores associados utilizando o IAC.

Levando em consideração que o IAC é um índice recente que pode ser utilizado para identificação da adiposidade corporal em estudos de base populacional e na prática clínica e das possíveis consequências do excesso de adiposidade corporal, este estudo objetivou verificar os fatores associados ao IAC em adultos, buscando contribuir com a avaliação da utilidade desse novo índice como indicador de adiposidade corporal.

\section{Materiais e métodos}

O presente estudo, transversal e de base populacional, faz parte das atividades do grupo de pesquisa sobre saúde e alimentação de Viçosa (ESA/ Viçosa), que conduziu um inquérito na área urbana da cidade de Viçosa de 2012 a 2014, sendo o objetivo geral estudar as condições de saúde da população adulta de 20 a 59 anos. Viçosa está localizada na região da Zona da Mata de Minas Gerais e caracteriza-se por ser uma cidade universitária. Segundo o Censo Demográfico de 201028, a população é de 72.220 habitantes, sendo 93,0\% 
moradores da zona urbana (67.305 indivíduos), e destes, 43.431 indivíduos (60,0\% da população total) encontram-se na faixa etária de referência do estudo.

O projeto foi submetido e aprovado pelo $\mathrm{Co}$ mitê de Ética em Pesquisa da Universidade Federal de Viçosa, seguindo a legislação brasileira para estudos em humanos. Todos os participantes assinaram o termo de consentimento livre e esclarecido em duas vias e os procedimentos para a realização do estudo foram descritos anteriormente ${ }^{29}$.

A amostra do estudo foi calculada utilizandose o programa Epi-Info, versão 3.5.2 ${ }^{\circledR}$. Adotou-se, para este estudo, a população de referência com base na estimativa de 2010 (43.431 indivíduos), nível de confiança de 95,0\%, prevalência esperada do excesso de adiposidade corporal de 23,4\% ${ }^{30}$, erro amostral previsto de 3,6 pontos percentuais, efeito do delineamento amostral (deff) igual a 1,5 , acréscimos de $10,0 \%$ para perdas estimadas e $10,0 \%$ para controle de fatores de confusão.

Após o calculo, obteve-se uma amostra de 946 indivíduos, por procedimentos de amostragem probabilística, por conglomerados e em dois estágios: setor censitário e domicílio. Foram realizadas 1229 entrevistas domiciliares, sendo descartados 265 indivíduos por não completarem os exames laboratoriais, totalizando uma amostra final de 964 indivíduos. Registra-se que não foi observada diferença estatística entre as características sociodemográficas da amostra original e a utilizada nesse estudo (dados não apresentados em tabela).

O número de setores censitários foi determinado seguindo-se recomendações descritas na literatura $^{31}$. Selecionou-se 30 setores dentro os 99 existentes na zona urbana de Viçosa, MG, sem reposição. Em seguida, identificaram-se os quarteirões, sendo sorteados quatro em cada um dos setores sorteados. Após essa etapa, as esquinas foram numeradas e procedeu-se o sorteio do ponto inicial para a coleta de dados no sentido horário a partir da esquina inicial.

Os domicílios visitados por pelo menos quatro vezes sem que o entrevistador localizasse a pessoa a ser entrevistada, incluída uma visita no final de semana e uma noturna, foram contabilizados como perdas. Aqueles indivíduos que, em um primeiro momento, recusaram-se a participar do estudo foram contatados pelos supervisores de campo e aqueles que mantiveram a não concordância em participar do estudo foram contabilizados como recusas. Além disso, foram excluídos do estudo as mulheres em gestação, indivíduos acamados ou impossibilitados para mensuração das medidas e aqueles que apresentaram alguma deficiência mental que impedisse de responder as questões do questionário.

Toda a coleta de dados foi conduzida por equipe de profissionais treinada para a aplicação dos questionários e as medidas antropométricas foram realizadas por um único avaliador com o objetivo de minimizar variações e manter a validade interna do estudo.

Os questionários foram aplicados nas residências dos indivíduos e os exames laboratoriais realizados no Laboratório de Estudos sobre Saúde e Alimentação (ESA) da Universidade Federal de Viçosa. O questionário estruturado era composto de 14 seções sendo utilizadas, para análise neste estudo, as seções 1 (dados sociodemográficos), 2 (classe econômica), 6 (tabagismo), 7 (estado de saúde), 8 (antecedentes de doenças) e 12 (tempo gasto com TV).

A variável dependente deste estudo foi o IAC, calculado a partir da equação ${ }^{16}$ :

$$
\mathrm{IAC}=\frac{\text { circunferência do quadril }}{\text { altura } \mathrm{x} \sqrt{\text { altura }}}-18
$$

Para estimar a prevalência de excesso de adiposidade corporal pelo IAC, utilizaram-se os seguintes pontos de corte: $25,0 \%$ para os homens e $35,0 \%$ para as mulheres ${ }^{32}$.

A circunferência do quadril foi medida com fita inelástica da marca Sanny ${ }^{\circledR}$, com precisão de $0.1 \mathrm{~cm}$, mensurada ao nível da extensão máxima posterior do glúteo em um plano horizontal. A estatura foi medida de forma direta, com estadiômetro da marca Welmy, com extensão de 2,5m e resolução de $0,1 \mathrm{~cm}$, estando o indivíduo ereto, descalço ou somente com meias, com os calcanhares unidos, em superfície lisa, plana e rígida, com os braços pendentes ao longo do corpo. A massa corporal foi obtida por meio de balança digital, da marca TANITA, modelo Ironmam BC-554 ${ }^{\circledR}$, com capacidade de $200 \mathrm{~kg}$ e precisão de $100 \mathrm{~g}$, com os voluntários utilizando o mínimo de roupa possível e sem sapatos. Todas as medidas antropométricas foram realizadas seguindo procedimentos já descritos ${ }^{29}$.

As variáveis independentes foram o sexo, a idade (20-29 anos, 30-39 anos, 40-49 anos e 5059 anos), a cor da pele (branca e não branca), a escolaridade em anos, o estado civil (sem companheiro e com companheiro) e o poder aquisitivo avaliado pelo questionário da Associação Brasileira de Empresas de Pesquisas ${ }^{33}$ e categorizado em nível alto (classes A1, A2, B1 e B2), médio (classes C1 e C2) e baixo (Classes D e E). 
As variáveis independentes relacionadas ao estilo de vida foram o tabagismo (não fumante, fumante e ex-fumante), tempo de tela em minutos (até 2 horas/dia, de 2 a 4 horas/dia, maior que 4 horas/dia) e o nível de atividade física avaliado pelo International Physical Activity Questionnaire (IPAq), versão longa, validado para a população brasileira ${ }^{34}$, sendo utilizado neste estudo o domínio 4 (atividades físicas de recreação, esporte, exercício e lazer). O nível de atividade física foi categorizado em inativo fisicamente $(<$ 150 minutos de atividades físicas nos últimos sete dias) e ativo fisicamente ( $\geq 150$ minutos de atividades físicas nos últimos sete dias), conforme recomendações descritas na literatura ${ }^{35}$.

Avaliou-se, também, a presença ou não de doenças autorreferidas (diabetes, hipertensão arterial e colesterol elevado), a autoavaliação de saúde (muito bom/bom e regular/ruim) e a satisfação com a imagem corporal (satisfeito e insatisfeito) mediante a escala de silhuetas para adultos, validada para a população brasileira ${ }^{36}$.

A variável dependente (IAC) foi analisada de forma contínua, atendendo a todos os pressupostos de normalidade. Realizou-se análise descritiva dos dados e utilizou-se o intervalo de confiança para analisar as diferenças entre as médias do IAC. Para verificar a associação entre o IAC e as variáveis independentes utilizou-se a regressão linear simples. As variáveis independentes que apresentaram associação com o desfecho $(\mathrm{p}<$ $0,20)$ foram tratadas como possíveis variáveis de confusão e avaliadas no modelo de regressão linear múltipla, sendo mantidas, no modelo final, apenas aquelas que se associaram ao desfecho com $\mathrm{p}<0,05$. Foram testadas interações entre as variáveis, não sendo nenhuma significativa. Foi avaliada a normalidade dos resíduos, sendo observado ajuste adequado na análise gráfica.

Os dados foram tabulados no software Epidata, em duplicata, e conferidos no módulo Data Compare. A análise dos dados foi realizada no aplicativo Stata, versão 13.1. Considerando a natureza complexa da amostra, empregou-se o conjunto de comandos "svy". Foram atribuídos pesos amostrais para as variáveis sexo, idade e escolaridade com o objetivo de permitir, dessa forma, extrapolar os resultados deste estudo para toda a população adulta do município de Viçosa.

\section{Resultados}

Foram analisados os dados de 964 indivíduos, sendo 431 do sexo masculino e 533 do sexo feminino, com média de idade de 35,8 anos (IC $95 \% 33,1-38,5$ ) e 39,3 (IC 95\% 37,3- 41,3), respectivamente. Os homens apresentaram valores médios e estaticamente maiores que as mulheres para a massa corporal $(77,6 \mathrm{Kg}$ para os homens e $65,1 \mathrm{Kg}$ para as mulheres, $\mathrm{p}<0,05)$ e a estatura $(1,70$ para os homens e 1,60 para as mulheres, $p$ $<0,05)$. Nas mulheres, os valores médios para a circunferência do quadril $(98,7$, para os homens e, 100,6, para as mulheres, $\mathrm{p}<0,05)$ e para o IAC ( $25,1 \%$, para os homens e, $31,8 \%$, para as mulheres, $\mathrm{p}<0,05)$ foram superiores estatisticamente quando comparados aos homens. A proporção de excesso de adiposidade corporal pelo IAC foi de 48,2\% (IC95\% 39,1-57,3) nos homens e de 25,6\% (IC95\% 19,6 - 32,7) nas mulheres.

$\mathrm{Na}$ Tabela 1, estão descritas as características sociodemográficas da amostra. Observa-se um aumento, em ambos os sexos, dos valores médios do IAC com a idade, naqueles que autoreferiram a cor não branca, nos indivíduos que vivem com companheiro e naqueles classificados com o nível socioeconômico baixo (classes D e E), sendo verificada significância estatística no aumento do IAC apenas nas variáveis idade ( $p<0,01)$, escolaridade ( $\mathrm{p}<0,01)$ e estado civil $(\mathrm{p}<0,01)$. Em relação à escolaridade, os maiores valores de IAC foram observados naqueles que relataram 5 a 8 anos de estudo, em ambos os sexos. Ao comparar os valores médios do IAC entre os sexos para as variáveis sociodemográficas, observou-se que no sexo feminino, como esperado, os valores foram significantemente superiores $(p<0,05)$ aos observados no sexo masculino, conforme descrito na Tabela 1.

Em relação às características comportamentais (Tabela 2), os valores médios do IAC foram significantemente superiores nos ex-fumantes ( $p$ $=0,03)$ e nos indivíduos inativos fisicamente ( $\mathrm{p}$ $<0,01$ ). Houve diferença estatística nos valores médios do IAC no nível de atividade física apenas no sexo masculino $(\mathrm{p}<0,05)$.

$\mathrm{Na}$ Tabela 3, percebe-se que as proporções de indivíduos com diabetes, hipertensão, colesterol elevado e autoavaliação da saúde ruim ou regular, em ambos os sexos, foram baixas. Porém, quanto à insatisfação com a imagem corporal, houve uma grande proporção de insatisfeitos tanto no sexo masculino (83,9\% IC95\% 78,9 $87,9)$ quanto no sexo feminino $(87,0 \%$ IC $95 \%$ $81,7-90,9)$. Os valores médios do IAC foram 
Tabela 1. Valores de média, regressão univariada e respectivos intervalos de confiança do índice de adiposidade corporal em adultos, segundo o sexo, para as variáveis socioeconômicas. Viçosa, MG, Brasil, 2012/2013. (n = 964)

\begin{tabular}{|c|c|c|c|c|c|}
\hline \multirow[b]{2}{*}{ Variáveis } & \multicolumn{2}{|c|}{ Masculino } & \multicolumn{2}{|c|}{ Feminino } & \multirow[b]{2}{*}{$\beta($ IC 95\%) } \\
\hline & $\begin{array}{l}\text { Proporção } \\
\text { (ep) }\end{array}$ & $\begin{array}{c}\text { IAC } \\
\text { Média (IC 95\%) } \\
\end{array}$ & $\begin{array}{l}\text { Proporção } \\
\text { (ep) }\end{array}$ & $\begin{array}{c}\text { IAC } \\
\text { Média (IC 95\%) }\end{array}$ & \\
\hline \multicolumn{6}{|l|}{ Idade em anos ${ }^{\star}$} \\
\hline $20-29$ & $37,0(6,1)$ & $23,6(23,2-24,0)$ & $24,9(3,6)$ & $29,0(28,3-29,8)$ & - \\
\hline $30-39$ & $25,5(3,2)$ & $24,9(24,2-25,7)$ & $24,6(2,0)$ & $32,1(31,0-33,2)$ & $2,7(1,9-3,4)$ \\
\hline $40-49$ & $18,9(3,3)$ & $26,8(25,7-27,9)$ & $26,7(3,6)$ & $32,9(31,8-34,2)$ & $4,6(3,7-5,6)$ \\
\hline $50-59$ & $18,6(3,1)$ & $26,8(25,9-27,6)$ & $23,8(2,7)$ & $33,4(32,0-34,7)$ & $4,7(3,4-6,0)$ \\
\hline \multicolumn{6}{|l|}{ Cor da pele e $^{*}$} \\
\hline Branca & $42,8(4,2)$ & $24,8(24,1-25,5)$ & $37,1(3,4)$ & $31,1(29,8-32,4)$ & - \\
\hline Não branca & $57,1(4,2)$ & $25,4(24,6-26,1)$ & $62,9(3,4)$ & $32,3(31,1-33,5)$ & $1,2(0,1-2,3)$ \\
\hline \multicolumn{6}{|l|}{ Escolaridade em anos ${ }^{*}$} \\
\hline$\geq 13$ & $51,2(7,5)$ & $24,3(23,9-24,8)$ & $42,1(5,7)$ & $30,3(29,3-31,2)$ & $-3,6(-5,8--1,3)$ \\
\hline $9-12$ & $20,4(3,2)$ & $25,7(24,4-26,9)$ & $20,6(1,6)$ & $31,5(30,6-32,4)$ & $-2,0(-4,0-0,1)$ \\
\hline $5-8$ & $14,5(2,9)$ & $26,4(25,8-27,1)$ & $16,0(2,2)$ & $34,2(33,3-35,3)$ & $-0,1(-2,2-2,1)$ \\
\hline $0-4$ & $14,0(5,1)$ & $25,9(24,3-24,8)$ & $21,3(4,1)$ & $33,6(31,0-36,2)$ & - \\
\hline \multicolumn{6}{|l|}{ Estado civil ${ }^{\star}$} \\
\hline Sem companheiro & $48,4(6,6)$ & $24,0(23,4-24,7)$ & $47,7(4,2)$ & $30,4(29,4-31,3)$ & \\
\hline Com companheiro & $51,5(6,6)$ & $26,1(25,4-26,9)$ & $52,3(4,2)$ & $33,2(32,2-34,3)$ & $2,5(1,8-3,3)$ \\
\hline \multicolumn{6}{|c|}{ Classificação socioeconômica* } \\
\hline Elevada (A e B) & $30,7(3,5)$ & $25,3(24,4-26,3)$ & $22,7(3,4)$ & $31,8(30,4-33,3)$ & - \\
\hline Intermediária $(\mathrm{C})$ & $64,9(3,5)$ & $25,0(24,4-25,6)$ & $66,9(3,0)$ & $31,8(30,7-33,0)$ & $0,4(-0,6-1,4)$ \\
\hline Baixa (D e E) & $4,5(1,8)$ & $25,8(23,0-28,7)$ & $10,4(2,2)$ & $32,2(30,2-34,1)$ & $2,2(0,1-4,3)$ \\
\hline
\end{tabular}

Tabela 2. Valores de média, regressão univariada e respectivos intervalos de confiança do índice de adiposidade corporal em adultos, segundo o sexo, para as variáveis comportamentais. Viçosa, MG, Brasil, 2012/2013. (n = 964).

\begin{tabular}{|c|c|c|c|c|c|}
\hline \multirow[b]{2}{*}{ Variáveis } & \multicolumn{2}{|c|}{ Masculino } & \multicolumn{2}{|c|}{ Feminino } & \multirow[b]{2}{*}{$\beta($ IC 95\%) } \\
\hline & $\begin{array}{c}\text { Proporção } \\
\text { (ep) }\end{array}$ & IAC (IC 95\%) & $\begin{array}{c}\text { Proporção } \\
\text { (ep) }\end{array}$ & IAC (IC 95\%) & \\
\hline \multicolumn{6}{|l|}{ Tabagismo* $^{\star}$} \\
\hline Não fumante & $61,6(4,5)$ & $24,6(24,1-25,2)$ & $69,5(3,1)$ & $31,6(30,4-32,5)$ & - \\
\hline Fumante & $17,1(3,0)$ & $24,3(23,5-25,0)$ & $11,0(1,8)$ & $31,7(30,1-33,3)$ & $-1,1(-2,3-0,1)$ \\
\hline Ex-fumante & $21,3(4,8)$ & $27,2(25,7-28,7)$ & $19,5(2,5)$ & $33,4(31,6-35,2)$ & $1,9(0,2-3,6)$ \\
\hline \multicolumn{6}{|c|}{ Tempo de tela (horas/dia)* } \\
\hline $0-2$ & $42,9(3,1)$ & $24,2(23,9-25,4)$ & $46,4(2,3)$ & $31,4(30,2-32,6)$ & - \\
\hline $2-4$ & $38,9(1,8)$ & $25,5(24,6-26,4)$ & $36,4(2,5)$ & $32,1(31,1-33,1)$ & $0,5(-0,6-1,6)$ \\
\hline$>4$ & $18,2(2,5)$ & $25,7(24,6-26,8)$ & $17,1(1,6)$ & $32,6(31,3-33,8)$ & $0,9(-0,1-1,8)$ \\
\hline \multicolumn{6}{|l|}{ Nível de atividade física* } \\
\hline Inativo fisicamente & $75,2(3,9)$ & $25,5(24,8-26,2)$ & $80,3(2,7)$ & $32,1(31,2-33,2)$ & - \\
\hline Ativo fisicamente & $24,8(3,9)$ & $24,0(23,4-24,7)$ & $19,8(2,7)$ & $30,7(29,2-32,2)$ & $-1,9(-2,9--1,0)$ \\
\hline
\end{tabular}

* variáveis incluídas na regressão linear multivariada por apresentarem valor de p $<0,20$.

maiores naqueles com diabetes ( $\mathrm{p}<0,01)$, hipertensão $(\mathrm{p}<0,01)$, colesterol elevado $(\mathrm{p}<0,01)$, saúde ruim ou irregular $(\mathrm{p}<0,01)$ e insatisfeitos com a imagem corporal ( $p<0,01)$, em ambos os sexos. Foram observadas diferenças significantes nos valores médios do IAC, para o sexo masculi- 
no, apenas nos indivíduos que autoreferiram ter hipertensão ( $\mathrm{p}<0,05)$, colesterol elevado ( $\mathrm{p}<$ $0,05)$ e insatisfeitos com a imagem corporal ( $\mathrm{p}<$ $0,05)$, enquanto no sexo feminino, foram observadas diferenças significantes na autoavaliação da saúde $(\mathrm{p}<0,05)$ e na satisfação com a imagem corporal $(\mathrm{p}<0,05)$ (Tabela 3).

Observa-se na Tabela 4, após ajuste para as variáveis de confusão, que as variáveis sexo, faixa etária, autoavaliação da saúde e satisfação com a

Tabela 3. Valores de média, regressão univariada e respectivos intervalos de confiança do índice de adiposidade corporal em adultos, segundo o sexo, para as variáveis de morbidades autoreferidas e de percepção corporal. Viçosa, MG, Brasil, 2012/2013. ( $\mathrm{n}=964)$

\begin{tabular}{|c|c|c|c|c|c|}
\hline \multirow[b]{2}{*}{ Variáveis } & \multicolumn{2}{|c|}{ Masculino } & \multicolumn{2}{|c|}{ Feminino } & \multirow[b]{2}{*}{$\beta($ IC 95\%) } \\
\hline & $\begin{array}{l}\text { Proporção } \\
\text { (ep) }\end{array}$ & IAC (IC 95\%) & $\begin{array}{l}\text { Proporção } \\
\text { (ep) }\end{array}$ & IAC (IC 95\%) & \\
\hline \multicolumn{6}{|l|}{ Diabetes ${ }^{*}$} \\
\hline Não & $95,4(1,6)$ & $25,1(24,4-25,7)$ & $93,9(1,5)$ & $31,6(30,7-32,5)$ & - \\
\hline Sim & $4,5(1,6)$ & $26,8(24,7-28,8)$ & $6,0(1,5)$ & $34,5(32,1-36,8)$ & $2,8(0,8-4,9)$ \\
\hline \multicolumn{6}{|l|}{ Hipertensão* } \\
\hline Não & $84,0(2,8)$ & $24,7(24,1-25,3)$ & $78,9(3,1)$ & $31,4(30,4-32,3)$ & - \\
\hline Sim & $16,0(2,8)$ & $27,3(25,4-29,3)$ & $21,1(3,1)$ & $33,6(32,0-35,2)$ & $2,9(1,9-4,0)$ \\
\hline \multicolumn{6}{|l|}{ Colesterol elevado* $^{*}$} \\
\hline Não & $84,3(2,0)$ & $24,8(24,2-25,4)$ & $73,4(3,1)$ & $31,4(30,4-32,4)$ & - \\
\hline Sim & $15,7(2,0)$ & $26,9(25,7-28,0)$ & $26,6(3,1)$ & $33,1(31,6-34,6)$ & $2,9(1,8-4,0)$ \\
\hline \multicolumn{6}{|c|}{ Autoavaliação de saúde ${ }^{*}$} \\
\hline Regular e ruim & $25,9(3,2)$ & $26,2(24,9-27,5)$ & $36,1(3,5)$ & $33,9(32,4-35,4)$ & - \\
\hline Muito bom e bom & $74,1(3,2)$ & $24,8(24,1-25,4)$ & $63,9(3,5)$ & $30,7(29,9-31,5)$ & $-3,2(-4,3--2,0)$ \\
\hline \multicolumn{6}{|c|}{ Satisfação com a imagem corporal ${ }^{*}$} \\
\hline Satisfeito & $16,0(2,2)$ & $23,4(22,6-24,5)$ & $13,0(2,2)$ & $28,9(28,0-30,0)$ & - \\
\hline Insatisfeito & $83,9(2,2)$ & $25,4(24,7-26,1)$ & $87,0(2,2)$ & $32,3(31,3-33,3)$ & $3,0(1,7-4,3)$ \\
\hline
\end{tabular}

Tabela 4. Modelo final da regressão múltipla para fatores associados ao índice de adiposidade corporal em adultos. Viçosa, MG, Brasil, 2012/2013. (n=964)

\begin{tabular}{|c|c|c|c|}
\hline Variável & Coeficiente $\boldsymbol{\beta}$ & IC $95 \%$ & $\mathbf{p}$ \\
\hline \multicolumn{4}{|l|}{ Sexo } \\
\hline Masculino & - & - & $<0,001$ \\
\hline Feminino & 6,2 & $5,4-7,0$ & \\
\hline Faixa etária (anos) & & & $<0,01$ \\
\hline $20-29$ & - & - & \\
\hline $30-39$ & 1,1 & $0,4-1,7$ & \\
\hline $40-49$ & 2,3 & $1,4-3,1$ & \\
\hline $50-59$ & 2,3 & $1,3-3,2$ & \\
\hline Estado civil & & & $<0,01$ \\
\hline Sem companheiro & - & - & \\
\hline Com companheiro & 1,3 & $0,2-2,0$ & \\
\hline Autoavaliação de saúde & & & $<0,01$ \\
\hline Regular e ruim & - & - & \\
\hline Muito boa e boa & $-1,6$ & $-2,4--0,7$ & \\
\hline Satisfação com a imagem corporal & & & $<0,01$ \\
\hline Satisfeito & - & - & \\
\hline Insatisfeito & 2,4 & $1,6-3,2$ & \\
\hline
\end{tabular}


imagem corporal mantiveram-se, independentemente, associadas ao IAC. Este modelo final explicou $48,1 \%$ da variabilidade do IAC.

Estiveram positiva e independentemente associadas ao aumento do IAC as seguintes variáveis: sexo feminino $(\mathrm{p}<0,01)$, faixas etárias de 30 a 39 anos $(\mathrm{p}<0,01), 40$ a 49 anos $(\mathrm{p}<0,01)$ e 50 a 59 anos $(\mathrm{p}<0,01)$, viver com companheiro $(\mathrm{p}<$ $0,05)$ e insatisfação com a imagem corporal ( $\mathrm{p}<$ $0,01)$. A autoavaliação da saúde como muito boa ou boa $(\mathrm{p}<0,05)$ esteve negativa e independentemente associada ao IAC.

\section{Discussão}

O IAC, descrito por Bergman et al. ${ }^{16}$, recentemente, foi proposto como uma alternativa simples para predizer a adiposidade corporal, visto que os métodos utilizados requerem o uso de equipamentos ou apresentam algumas limitações para predizer a adiposidade corporal como, por exemplo o IMC ${ }^{12-15}$. A validade do IAC está bem documentada na literatura ${ }^{13,16-27,37}$, porém estudos da prevalência do excesso de adiposidade corporal utilizando o IAC e fatores associados não foram identificados, até o presente momento. Este é o primeiro estudo, de que se tem conhecimento, que se concentrou em identificar a prevalência de adiposidade corporal em adultos pelo IAC e associá-lo a fatores sociodemográficos, comportamentais, de morbidades autorreferidas e de percepção. Devido à ausência de estudos com esses objetivos, torna-se limitada a comparação dos resultados obtidos com trabalhos que empregaram a mesma metodologia.

A proporção elevada de excesso de adiposidade corporal pelo IAC nos homens (48,2\% IC95\% $39,1-57,3)$ e nas mulheres (25,6\% IC95\% 19,6 - 32,7) é um dado importante que corrobora com a perspectiva do aumento da obesidade em todo mundo ${ }^{1}$. Porém, esses valores são superiores àqueles observados em adultos brasileiros, quando se utiliza outros indicadores de excesso de adiposidade corporal, para o sexo masculino $\mathrm{o}^{30,38-40} \mathrm{e}$ inferiores no sexo feminino ${ }^{30,39}$. Em adultos do sexo masculino de Bogotá, na Colombia ${ }^{22}$, a prevalência de obesidade pelo IAC $(50,1 \%)$ foi similar àquela observada em nosso estudo (48,2\%). Essas variações da prevalência de excesso de adiposidade corporal pelo IAC quando comparados a outras técnicas, talvez estejam relacionadas à utilização de diferentes métodos de avaliação do excesso de adiposidade corporal ou às características distintas das populações avaliadas. Outro ponto importante e que pode justificar essa diferença nas proporções é a superestimação observada no sexo masculino ${ }^{13,22,24}$ e a subestimação no sexo feminino ao predizer a adiposidade corporal com o $\mathrm{IAC}^{13,24}$. Apesar disso, destaca-se que todas as medidas antropométricas utilizadas neste estudo foram realizadas por um único avaliador e com equipamentos calibrados, o que confere um rigor metodológico e reforçam sua validade interna, sendo que nos parece que essa alta prevalência não foi decorrente de erros sistemáticos.

Após análise univariada, as variáveis sexo, faixa etária, estado civil, autoavaliação da saúde e insatisfação com a imagem corporal mantiveram-se associadas ao IAC. Resultados semelhantes foram observados para a associação do excesso de adiposidade corporal, independente do indicador utilizado, com o sexo ${ }^{39,40}$, a faixa etária $^{39,41,42}$, o estado civil ${ }^{40}$, a autoavaliação da saúde $^{43}$ e a insatisfação com a imagem corporal ${ }^{44}$.

No presente estudo, observou-se que no sexo feminino há um aumento médio de $6,2 \%$ no IAC em relação ao sexo masculino. Esse excesso de adiposidade corporal no sexo feminino quando comparado ao sexo masculino, independente do método utilizado, também foi observado em mulheres de Salvador que apresentaram uma prevalência 2,7 vezes mais elevada de excesso de gordura abdominal quando comparada aos homens ${ }^{41}$. Este incremento no sexo feminino pode estar relacionado às atividades laborais exercidas pelos indivíduos do sexo masculino que, culturalmente, desempenham atividades com um maior dispêndio energético ${ }^{40}$. Outro aspecto importante é acumulo de gordura subcutânea que acontece nas mulheres em função do envelhecimento e redistribuição da gordura ${ }^{32}$.

Também foi observada uma associação positiva entre a adiposidade corporal e idade, com aumento médio de, aproximadamente, 1,7\% em cada categoria de idade. Torna-se importante destacar que há evidencias na literatura demonstrando que a relação entre a adiposidade e mortalidade pode variar em função do aumento da idade $^{45-47}$, o que reforça a necessidade de verificarmos o excesso de adiposidade corporal em relação à faixa etária dos indivíduos. Em uma coorte com 77.505 mulheres pós-menopausa, Thomson et al. ${ }^{48}$ encontraram valores médios para o IAC $(32,8 \mathrm{dp} 6,4)$, na faixa etária de 50 a 59 anos, similares aos observados neste estudo $(33,4$ dp 6,9). Estudo utilizando a razão cintura-estatura $^{40}$, como indicador de adiposidade corporal, também observou um aumento da adiposidade com a idade. O aumento da obesidade abdomi- 
nal também está associado ao avançar da idade em adultos, sendo observado um incremento de $11 \mathrm{~cm}$ no perímetro abdominal para cada mudança no estrato etário ${ }^{42}$. Uma explicação plausível para o aumento da adiposidade corporal com a idade pode estar na mudança do estilo de vida, com diminuição dos níveis de atividade física e, consequentemente, com a diminuição do metabolismo basal.

O aumento do IAC entre os casados corrobora com outros estudos descritos na literatura ${ }^{40,42}$. É possível que esse aumento da adiposidade corporal em indivíduos casados ou que vivem com companheiros seja decorrente do aumento na preocupação com a casa e os filhos o que pode diminuir o tempo livre para à pratica de atividade física geral, por gastarem mais tempo cuidando da família ${ }^{40}$. Além disso, a preocupação com o corpo talvez seja mais frequente naqueles indivíduos que vivem sem companheiro, devido a padrões impostos pela sociedade, o que pode contribuir para um maior cuidado com o excesso de adiposidade corporal.

O excesso de adiposidade corporal, identificado pelo IAC, demonstrou ser um fator importante para a autoavaliação da saúde em nosso estudo. Os indivíduos que autoreferiram ter uma saúde boa ou muito boa teve uma diminuição média de $-1,6 \%$ na adiposidade corporal quando comparados aqueles com saúde regular ou ruim. Assim como em nosso estudo, ao avaliarem 12.324 indivíduos com idade superior a 20 anos em todas as regiões do país, Pavão et al..$^{43}$ observaram que o excesso de adiposidade corporal se manteve associado a autoavaliação da saúde, sendo os obesos mais propensos a autoavaliarem sua saúde como ruim. A autoavaliação negativa da saúde apresenta um risco relativo de morte duas vezes maior em relação a autoavaliação da saúde positiva ${ }^{49}$, além disso pode ser um fator determinante na decisão de procurar um médico ou serviço especializado $^{43}$. Esses aspectos podem aumentar em indivíduos com excesso de adiposidade corporal, elevando a demanda dos serviços de saúde.

Em nosso estudo, observou-se que aqueles insatisfeitos com a imagem corporal apresentaram um incremento de $2,4 \%$ na adiposidade corporal. Ao analisar a insatisfação com a imagem corpo- ral em 500 mulheres iranianas, Nikniaz et al. ${ }^{50}$ observaram um aumento na insatisfação com a imagem corporal média ao aumentar o IMC ( $\mathrm{p}$ $<0,01$ ). Estudos realizados na população adulta brasileira $^{44} \mathrm{e}$ com universitários brasileiros ${ }^{51}$, também observaram associação entre indicadores de excesso de adiposidade corporal e insatisfação com a imagem corporal. A associação entre a adiposidade corporal e a insatisfação com a imagem corporal, observada neste estudo, talvez esteja associada à exagerada preocupação com a estética corporal imposta pela sociedade e pela mídia, provocando uma dicotomia entre o padrão de beleza imposto e o considerado adequado ${ }^{51}$, criando uma pressão que leva à internalização de um corpo ideal e um forte desejo para atingi-lo $\mathrm{o}^{52}$.

Este foi o primeiro estudo, que se tem conhecimento, a verificar a associação da adiposidade corporal estimada pelo IAC, mas apresenta algumas limitações que devem ser destacadas. A primeira delas é o delineamento transversal que não permite estabelecer uma relação temporal entre a exposição e o desfecho, porém este não era o objetivo de nosso estudo. Outro aspecto importante foi que a proporção de indivíduos selecionados não correspondeu aos dados referentes à população de Viçosa para o sexo, escolaridade e idade, sendo essa limitação corrigida a partir da ponderação da amostra. Como pontos fortes, destaca-se a utilização de questionários validados, o treinamento e controle de qualidade efetiva antes e durante a coleta de dados, além da utilização de um único avaliador para realização das medidas antropométricas, contribuindo, dessa forma, para validade interna deste estudo.

Em conclusão, os resultados deste estudo indicam que o sexo, idade, estado civil, autoavaliação da saúde e a insatisfação com a imagem corporal foram fatores determinantes para o aumento da adiposidade corporal, nessa população. Sugere-se a realização de novos estudos, principalmente com a população adolescente e idosa para verificar a associação do IAC com variáveis sociodemográficas, comportamentais e de percepção. Além disso, intervenções devem ser realizadas com o objetivo de diminuir os fatores de risco para o aumento da adiposidade corporal e, consequentemente, o aparecimento de comorbidades. 


\section{Colaboradores}

W Segheto: participou da concepção, delineamento e análise/interpretação dos dados, da redação do artigo e da aprovação da versão a ser publicada, bem como da coleta de dados. PC Hallal: participou da análise/interpretação dos dados, da redação do artigo, da revisão critica e da aprovação da versão a ser publicada. JCB Marins: participou da análise/interpretação dos dados, da redação do artigo, da revisão critica e da aprovação da versão a ser publicada. DCG Silva: participou da concepção e delineamento do estudo, da redação do artigo e da aprovação da versão a ser publicada, bem como da coleta de dados. FA Coelho: participou da concepção e delineamento do estudo, da redação do artigo e da aprovação da versão a ser publicada, bem como da coleta de dados. AQ Ribeiro: participou da análise/interpretação dos dados, da redação do artigo, da revisão critica e da aprovação da versão a ser pu- blicada. SHO Morais: participou da concepção e delineamento do estudo, da redação do artigo e da aprovação da versão a ser publicada, bem como da coleta de dados. GZ Longo: participou da concepção, delineamento e análise/interpretação dos dados, da redação do artigo e da revisão critica para aprovação da versão a ser publicada e coordenou a coleta de dados.

\section{Agradecimentos}

Os autores agradecem a todos os voluntários que contribuíram com o estudo, aos bolsistas de iniciação científica e voluntários que participaram da coleta de dados, ao Conselho Nacional de Desenvolvimento Científico e Tecnológico (CNPq) e a Fundação de Amparo à Pesquisa do estado de Minas Gerais (FAPEMIG) pelo apoio financeiro para o desenvolvimento da pesquisa.

\section{Referências}

1. World Health Organization (WHO). Obesity and overweight [Internet]. 2015. [acessado 2015 Abr 22]. Disponível em: http://www.who.int/mediacentre /factsheets/ fs $311 /$ en/

2. Apovian CM, Gokce N. Obesity and Cardiovascular Disease. Circulation 2012; 125(9):1178-1182.

3. Kahn SE, Cooper ME, Del Prato S. Pathophysiology and treatment of type 2 diabetes: perspectives on the past, present, and future. Lancet 2014; 383(9922):10681083.

4. Jordan AS, McSharry DG, Malhotra A. Adult obstructive sleep apnoea. Lancet 2014; 383(9922):736-747.

5. Catalán V, Gómez-Ambrosi J, Rodríguez A, Frühbeck G. Adipose tissue immunity and cancer. Front Physiol 2013; 4:275

6. Withrow D, Alter DA. The economic burden of obesity worldwide: a systematic review of the direct costs of obesity. Obes Rev 2011; 12(2):131-141.

7. Tobias D, Pan A, Hu F. BMI and Mortality among Adults with Incident Type 2 Diabetes. $N$ Engl J Med 2014; 370(14):1361-1364.
8. Finkelstein EA, Trogdon JG, Cohen JW, Dietz W. Annual Medical Spending Attributable To Obesity: Payer-And Service-Specific Estimates. Health Aff 2009; 28(5):w822-831.

9. Mazzoccante RP, Moraes JFVN, Campbell CSG. Gastos públicos diretos com a obesidade e doenças associadas no brasil. Rev Ciências Médicas 2012; 21(1-6):25-34.

10. Ellis KJ. Human Body Composition: In Vivo Methods. Physiol Rev 2000; 80(2):649- 80.

11. Guedes DP. Procedimentos clínicos utilizados para análise da composição corporal. Rev Bras Cineantropometria e Desempenho Hum. 2013; 15(1):113-129.

12. Piers LS, Rowley KG, Soares MJ, O’Dea K. Relation of adiposity and body fat distribution to body mass index in Australians of Aboriginal and European ancestry. Eur J Clin Nutr 2003; 57(8):956-963.

13. López AA, Cespedes ML, Vicente T, Tomas M, Bennasar-Veny M, Tauler P, Aquilo A. Body Adiposity Index Utilization in a Spanish Mediterranean Population: Comparison with the Body Mass Index. PLoS One 2012; 7(4):e35281. 
14. Rahman M, Berenson AB. Accuracy of Current Body Mass Index Obesity Classification for White, Black, and Hispanic Reproductive-Age Women. Obstet Gynecol 2010; 115(5):982-988.

15. Garrido-Chamorro R, Sirvent-Belando J, Gonzalez-Lorenzo M, Martin-Carratala M, Roche E. Correlation between body mass index and body composition in elite athletes. J Sports Med Phys Fitness 2009; 49(3):278-284.

16. Bergman RN, Stefanovski D, Buchanan TA, Sumner AE, Reynolds JC, Sebring NG, Xiang AH, Watanabe RM. A Better Index of Body Adiposity. Obesity 2011; 19(5):1083-1089.

17. Godoy-Matos AF, Moreira RO, Valerio CM, Mory PB, Moises RS. A New Method for Body Fat Evaluation, Body Adiposity Index, Is Useful in Women With Familial Partial Lipodystrophy. Obesity 2012; 20(2):440-443.

18. Chang H, Simonsick EM, Ferrucci L, Cooper JA. Validation Study of the Body Adiposity Index as a Predictor of Percent Body Fat in Older Individuals: Findings From the BLSA. Journals Gerontol Ser A Biol Sci Med Sci 2014; 69(9):1069-1075.

19. Johnson W, Chumlea WC, Czerwinski SA, Demerath EW. Concordance of the Recently Published Body Adiposity Index With Measured Body Fat Percent in EuropeanAmerican Adults. Obesity 2012; 20(4):900-903.

20. Alvim RO, Mourao-Junior CA, Oliveira CM, Krieger JE, Mill JG, Pereira AC. Body Mass Index, Waist Circumference, Body Adiposity Index, and Risk for Type 2 Diabetes in Two Populations in Brazil: General and Amerindian. PLoS One 2014; 9(6):e100223.

21. García AI, Niño-Silva LA, González-Ruíz K, RamírezVélez R. Utilidad del índice de adiposidad corporal como indicador de obesidad y predictor de riesgo cardiovascular en adultos de Bogotá, Colombia. Endocrinol y Nutr 2015; 62(3):130-137.

22. González-Ruíz K, Correa-Bautista JE, Ramírez-Vélez R. Evaluation of the body adiposity index in predicting percentage body fat among Colombian adults. Nutr Hosp. 2015; 32(1):55-60.

23. González-Ruíz K, Correa-Bautista JE, Ramírez-Vélez R. Body adiposity and its relationship of metabolic syndrome components in Colombian adults. Nutr Hosp 2015; 32(4):1468-1475.

24. Carpio Rivera E, Hernández-Elizondo J, Salicetti-Fonseca A, Solera-Herrera A, Moncada-Jiménez J. Predictive validity of the body adiposity index in Costa Rican students. Am J Hum Biol 2015; 28(3):394-397.

25. Silva MIB, Vale BS, Lemos CCS, Torres MRSG, Bregman R. Body adiposity index assess body fat with high accuracy in nondialyzed chronic kidney disease patients. Obesity 2013; 21(3):546-552.

26. Belarmino G, Horie LM,Sala PC, Torrinhas RS, Heymsfield SB, Waitzberg DL. Body adiposity index performance in estimating body fat in sample of severely obese Brazilian patients. Nutr J 2015; 14(1):130.

27. Cerqueira M, Amorim P, Magalhães F, Castro E, Franco F, Franceschini S, Cerqueira L, Marins J, Doimo L. Validity of body adiposity index in predicting body fat in a sample of Brazilian women. Obesity 2013; 21(12):E696-699.

28. Instituto Brasileiro de Geografia e Estatística (IBGE). Censo Demográfico 2010. Características da população e dos domicílios. Rio de Janeiro: IBGE; 2011.
29. Segheto W, Silva DCG, Coelho FA, Reis VG, Morais SHO, Marins JCB, Ribeiro AQ, Longo GZ. Body adiposity index and associated factors in adults: method and logistics of a population based study. Nutr Hosp 2015; 32(1):101-109.

30. Longo GZ, Neves J, Castro TG, Pedroso MRO, Matos IB. Prevalência e distribuição dos fatores de risco para doenças crônicas não transmissíveis entre adultos da cidade de Lages (SC), Sul do Brasil, 2007. Rev Bras Epidemiol 2011; 14(4):698-708.

31. Barros FC, Victora CG. Epidemiologia da Saúde Infantil. Um manual para diagnósticos comunitários. São Paulo: Hucitec, UNICEF; 1998.

32. World Health Organization (WHO). Physical status: the use and interpretation of anthropometry. Report of a WHO Expert Committee. Geneva: WHO; 1995.

33. Associação Brasileira de Empresas de Pesquisa (ABEP). Critério de classificação econômica Brasil - Dados com base no levantamento sócio econômico. São Paulo: ABEP; 2012.

34. Pardini R, Matsudo S, Araújo T, Matsudo V, Andrade E, Braggion G, Andrade D, Oliveira L, Figueira A, Raso V. Validação do questionário internacional de nível de atividade física (IPAQ - versão 6):estudo piloto em adultos jovens brasileiros. Rev Bras Ciência e Mov 2001; 9(3):45-51.

35. Haskell WL, Lee I-M, Pate RR, Powell KE, Blair SN, Franklin BA, Macera CA, Heath GW, Thompson PD, Bauman A. Physical Activity and Public Health: updated recommendation for adults from the American College of Sports Medicine and the American Heart Association. Med Sci Sport Exerc 2007; 39(8):14231434.

36. Kakeshita IS, Silva AIP, Zanatta DP, Almeida SS. Construção e fidedignidade teste-reteste de escalas de silhuetas brasileiras para adultos e crianças. Psicol Teor e Pesqui 2009; 25(2):263-270.

37. Sun G, Cahill F, Gulliver W, Yi Y, Xie Y, Bridger T, Pace D, Zhang H. Concordance of BAI and BMI with DXA in the Newfoundland Population. Obesity 2013; 21(3):499-503.

38. Brasil. Ministério da Saúde (MS). Vigitel Brasil 2011: Vigilância de Fatores de Risco e Proteção para Doenças Crônicas por Inquérito Telefônico. Brasília: MS; 2012.

39. Pinho CPS, Diniz AS, Arruda IKG, Filho MB, Coelho PC, Sequeira LAS, Lira PIC. Prevalência e fatores associados à obesidade abdominal em indivíduos na faixa etária de 25 a 59 anos do Estado de Pernambuco, Brasil. Cad Saude Publica 2013; 29(2):313-324.

40. Soares DA, Barreto SM. Sobrepeso e obesidade abdominal em adultos quilombolas, Bahia, Brasil. Cad Saude Publica 2014; 30(2):341-354.

41. Oliveira LPM, Assis AMO, Silva MCM, Santana MLP, Santos NS, Pinheiro SMC, Barreto ML, Souza CO. Fatores associados a excesso de peso e concentração de gordura abdominal em adultos na cidade de Salvador, Bahia, Brasil. Cad Saude Publica 2009; 25(3):570-582.

42. Longo GZ, Segheto W, Silva DCG, Ribeiro AQ, Franco FS, Souza JD, Coelho FA, Oliveira JM. Abdominal perimeter is associated with food intake, sociodemographic and behavioral factors among adults in southern Brazil: a population-based study. Nutr Hosp 2015; 31(2):621-628. 
43. Pavão ALB, Werneck GL, Campos MR. Autoavaliação do estado de saúde e a associação com fatores sociodemográficos, hábitos de vida e morbidade na população: um inquérito nacional. Cad Saude Publica 2013; 29(4):723-734.

44. Silva DAS, Nahas MV, Sousa TF, Del Duca GF, Peres KG. Prevalence and associated factors with body image dissatisfaction among adults in southern Brazil: A populationbased study. Body Image 2011; 8(4):427-431.

45. Zajacova A, Ailshire J. Body mass trajectories and mortality among older adults: a joint growth mixture-discrete-time survival analysis. Gerontologist 2014; 54(2):221-231.

46. Zheng H, Tumin D, Qian Z. Obesity and mortality risk: new findings from body mass index trajectories. $\mathrm{Am} J$ Epidemiol 2013; 178(11):1591-1599.

47. Botoseneanu A, Liang J. Latent heterogeneity in longterm trajectories of body mass index in older adults. $J$ Aging Health 2013; 25(2):342-363.

48. Thomson CA, Garcia DO, Wertheim BC, Hingle MD, Bea JW, Zaslavsky O, Caire-Juvera G, Rohan T, Vitolins MZ, Thompson PA, Lewis CE. Body shape, adiposity index, and mortality in postmenopausal women: Findings from the Women's Health Initiative. Obesity (silver Spring) 2016; 0(0):1-9.

49. DeSalvo KB, Bloser N, Reynolds K, He J, Muntner P. Mortality prediction with a single general self-rated health question. J Gen Intern Med 2006; 21(3):267-275.

50. Nikniaz Z, Mahdavi R, Amiri S, Ostadrahimi A, Nikniaz L. Factors associated with body image dissatisfaction and distortion among Iranian women. Eat Behav 2016; 22(2016):5-9.

51. Quadros TMB, Gordia AP, Martins CR, Silva DAS, Ferrari EP, Petroski EL. Imagem corporal em universitários: associação com estado nutricional e sexo. Motriz 2010; 16(1):78-85.

52. Stefanile C, Matera, C, Pisani E, Zambrini I. Insoddisfazione corporea in adolescenza: influenze di fattori bio-psico-sociali. Psicologia della Salute 2009; 2:51-65. 
